\title{
Author Correction: Impact of high-speed rail on road traffic and greenhouse gas emissions
}

Yatang Lin, Yu Qin iD, Jing Wu and Mandi Xu

Correction to: Nature Climate Change https://doi.org/10.1038/s41558-021-01190-8, published online 25 October 2021

In the version of this Article initially published, there were errors in the main text, Fig. 3, Tables 2 and 3, equation (3) in Methods and the Supplementary Information owing to a conversion error from log-point to actual growth rate when quantifying the reduction of vehicle volume on highways. We show that high-speed rail connection leads to a $20.5 \log$-point reduction in passenger vehicles and 15.7 $\log$-points reduction in freight vehicles on highways, and we use these numbers to calculate the implications on greenhouse gas emissions. When we transform $\log$ points to growth rate, we should have used $\mathrm{e}^{(-0.205)}-1$ and $\mathrm{e}^{(-0.157)}-1$, but we incorrectly used $1-\mathrm{e}^{(0.205)}$ and $1-\mathrm{e}^{(0.157)}$. As a consequence, the emission reduction reported from high-speed rail was -14.76 million tons rather than the correct -11.18 million tons. Corrected calculations are discussed below.

In the abstract, in the sentence beginning "These effects translate into an annual reduction of...," the terms " 14.76 " and " $1.75 \%$ " have been corrected to " 11.183 " and " $1.33 \%$," respectively. In the introduction section, in the sentence beginning "We find the HSR connection reduces...," "14.758" and " $1.75 \%$ " have been corrected to " 11.183 " and "1.33\%," respectively. In the "Impact on GHG emissions by replacing road traffic" section, in the sentence beginning "Compared with the pre-HSR situation...," "14.758" has been corrected to read "11.183"; in the sentence starting with "The estimates range from...," "7.738," "16.966," "14.758" and "1.75\%" have been corrected to read "5.574," "12.946," "11.183" and "1.33\%," respectively. In the sentence beginning "We document that...," "4.241" and "18.999" have been corrected to read " 3.354 " and " 14.537 ," respectively. In the sentence beginning "Among them...," "3.176" has been corrected to read "2.392"; in the sentence beginning "The overall GHG-emission reduction almost doubles...", "14.758," "26.239," and "3\%" have been corrected to read "11.183," "20.251," and "2.40\%," respectively. In the "Overall impact on traffic flows and GHG emissions" section, in the sentence beginning "After the connection of HSR..., "-13.705" and " -0.331 " have been corrected to read " -9.953 " and "2.002," respectively. In the "Conclusion" section, in the sentence beginning "The effects translate...," "1.75\%" has been corrected to read "1.33\%." In the "GHG-emission calculation" section of the Methods, in the final term of equation (3), " $\left(e^{\beta_{h}}-1\right)$ " have been corrected to read " $\left(1-e^{\beta_{h}}\right)$ "”

In the online versions of the article, relevant numbers in Tables 2 and 3, Fig. 3, Supplementary Note and Supplementary Tables 6-8, 10,16 , and 18 have been replaced to correct erroneous results caused by the same conversion error from log-point to the actual growth rate. Detailed comparisons of the Figure, Table, Supplementary Note and Supplementary Table changes are shown in the Supplementary Information file accompanying this amendment. The main article Supplementary Information has been replaced.

Supplementary information is available online.

Additional information

Supplementary information The online version contains supplementary material available at https://doi.org/10.1038/s41558-021-01253-w.

Published online: 8 December 2021

https://doi.org/10.1038/s41558-021-01253-w

(c) The Author(s), under exclusive licence to Springer Nature Limited 2022 